\title{
Errata: In situ permeation study of drug through the stratum corneum using attenuated total reflection Fourier transform infrared spectroscopic imaging
}

Jean-Michel Andanson

Imperial College London

Department of Chemical Engineering

South Kensington Campus

London SW7 2AZ

United Kingdom

Jonathan Hadgraft

University of London

The School of Pharmacy

29-39 Brunswick Square

London WC1N $1 \mathrm{AX}$

United Kingdom

Sergei G. Kazarian

Imperial College London

Department of Chemical Engineering

South Kensington Campus

London SW7 2AZ

United Kingdom

[DOI: $10.1117 / 1.3157197]$

This article [J. Biomed. Opt. 14, 034011 (May/June 2009)] was originally published online on 12 May 2009 with errors in the title, ninth line of the abstract, and the keyword list. The word reflectance has been replaced with the word reflection. All online versions of the article were corrected on 19 May 2009 and the article appears correctly in print. 